[Agr. Biol. Chem., Vol. 32, No. 10, p. 1261 1269, 1968]

\title{
A Comparison of the Structure of Curdlan and Pachyman
}

\author{
By Hiroshi Saito, Akira Mrsaki and Tokuya Harada \\ Institute of Scientific and Industrial Research, Osaka University, \\ Suita, Osaka \\ Received May 18, 1968
}

\begin{abstract}
The fine structure of curdlan produced by Alcaligenes faecalis var. myxogenes 10C3, mutant K, and pachyman from Poria cocos were investigated, with regard to their gel-forming properties. Periodate oxidation showed that both polysaccharides contain very high proportions of $(1 \rightarrow 3)$-linked glucose residues. On complete hydrolysis the glucan polyalcohol obtained by periodate oxidation and borohydride reduction of curdlan ( $\overline{\mathrm{DP}} 455)$ gave glucose and glycerol, in the molar ratio of $125 \sim 130: 1$, and on mild acid hydrolysis yielded degraded polysaccharide ( $\overline{\mathrm{DP}} 155)$, confirming the previous conclusion that curdlan has an essentially unbranched structure though it may contain a few internal $(1 \rightarrow 6)$ glucosidic linkages. On complete hydrolysis the glucan polyalcohol derived from pachyman (DP 255) gave glucose and glycerol, in the ratio of $40: 1$, and on mild hydrolysis it yielded degraded polysaccharide ( $\overline{\mathrm{DP}} 130)$. This indicates that pachyman contains on the average four branch points and one internal $(1 \rightarrow 6)$-glucosidic linkage in the molecule.
\end{abstract}

$\beta-1,3$ Glucans occur in yeasts, fungi and higher plants as structural constituents of cell walls or as reserve polysaccharides.

Our recent investigations ${ }^{1 \sim 31}$ showed that Alcaligenes faecalis var. myxogenes $10 \mathrm{C} 3$, mutant $\mathrm{K}$, produces a water-insoluble polysaccharide, culdlan, which is the first known bacterial $\beta-1,3$ glucan, as far as we are aware. Methylation and periodate oxidation studies ${ }^{4 \prime}$ indicated that curdlan is an essentially linear polysaccharide consisting mainly of $\beta-(1 \rightarrow 3)$ linked D-glucose residues, and may be closely related in chemical nature to pachyman from Poria $\operatorname{cocos}^{6}$ and paramylon from various sources. However, our preliminary study showed that the gel formed from pachyman

1) T. Harada, M. Masada, K. Fujimori and $I$. Maeda, Agr. Biol. Chem., 30, 196 (1966).

2) T. Harada, M. Masada, H. Hidaka and M.

Takada, J. Ferment. Technol., 44, 20 (1966).

3) T. Harada, K. Fujimori, S. Hirose and M Masada, Agr. Biol. Chem., 30, 764 (1966).

4) T. Harada, A. Misaki and H. Saito, Arch. Biochem. Biophys., 124, 292 (1968)

5) S. A. Warsi and W.J. Whelan, Chem. Ind. (London), 1957, 1573. by heat treatment is less resilient than the gel formed by heating curdlan, in aqueous suspension at $100^{\circ} \mathrm{C}$ for a few minutes. The latter gel is firm and resilient, as shown in the previous study. ${ }^{6}$ It was thought that this rheological difference might be due to the presence of anomalous linkages in the pachyman molecule. Accordingly, we compared the fine structure of curdlan and pachyman.

The present paper is on the significance of the structural difference between two polysaccharides, revealed by the Smith degradation procedure which involves periodate oxidation, borohydride reduction and acid hydrolysis."

\section{MATERIALS AND METHODS}

General methods. All evaporations were conducted under reduced pressure at $35 \sim 40^{\circ} \mathrm{C}$, unless otherwise stated.

6) I. Maeda, H. Saito, M. Masada, A. Misaki and T. Harada, Agr. Biol. Chem., 31, 1184 (1967)

7) I. J. Goldstein, G. W. Hay, B. A. Lewis and F. Smith, Abstr. paper Am. Chem. Soc., 135, 3D (1959). 
Paper chromatography was performed by the descending method on Toyoroshi No. 51 paper with the following solvent systems; (A) butanol-acetic acid-water $(4: 1: 5 \mathrm{v} / \mathrm{v})$, and (B) butanol-pyridinewater $(6: 4: 3 \mathrm{v} / \mathrm{v})$.

Sugars and sugar alcohols on the chromatograms were detected with alkaline silver nitrate by the method of Trevelyan et al.81 Reducing sugars were also detected by spraying the paper with $p$-anisidine hydrochloride in water-saturated butanol.

The glucose content of the polysaccharide preparations was estimated with phenol-sulfuric acid reagent.9) Glycerol and formaldehyde were determined by the chromotropic acid method,10) as modified by Hanahan and Olley.111

Periodate oxidation. To minimize overoxidation, curdlan and pachyman were oxidized with $0.01 \mathrm{M}$ sodium periodate at $3^{\circ} \mathrm{C}$ in the dark. Under these conditions hydrolysis of the formylester formed at the reducing end is very slow, 12) so that a linear chain of $(1 \rightarrow 3)$-linked glucose residues liberates 1 mole of formic acid from the non-reducing ends. The consumption of periodate and production of formic acid were determined by the usual arsenite method and titration with $0.01 \mathrm{~N}$ sodium hydroxide, respectively, and extrapolation of the linear portion of the graphs to zero time. Results were expressed as moles per anhydroglucose unit.

Measurement of gel strength. Ten $\mathrm{ml}$ aliquots of aqueous suspensions of various concentrations of curdlan and pachyman were homogenized in a Potter apparatus in the cold and then heated at $90^{\circ} \mathrm{C}$ for $10 \mathrm{~min}$ to give transparent gels. After cooling to $30^{\circ} \mathrm{C}$, the strength of the gel was measured with a curd meter (Iio Electric Co., type M301A).

Infrared analyses. Infrared spectra were measured in a $\mathrm{KBr}$ disk with a Hitachi Infrared Spectrophotomether, model Epl-2.

B-1,3 Glucanase. Basidiomycete QM806 and Rhizopus arrhizus QM1032, which produce exo- and endo$\beta-1,3$ glucanase, respectively, were grown in the

8) W. E. Trevelyan, D. P. Procter and J.S. Harrison, Nature, 166, 444 (1950).

9) M. Dubois, K. A. Gilles, J. K. Hamilton, P. A. Rebers and F. Smith, Anal. Chem., 28, 350 (1956).

10) M. Lambert and A. C. Neish, Can. J. Res., 28B, 83 (1950).

11) D. J. Hanahan and J. N. Olley, J. Biol. Chem., 231, 913 (1958).

12) F. B. Anderson, E. L. Hirst, D. J. Manners and A. G. Ross, J. Chem. Soc., 1958, 3233.
$0.5 \%$ starch medium of Reese and Mandels. ${ }^{131} \mathrm{Th}$ culture filtrates were evaporated to small volume in vacuo at $30^{\circ} \mathrm{C}$, and the crude enzymes were pri cipitated from them by addition of 2 volumes 0 cold acetone $\left(-10^{\circ} \mathrm{C}\right)$. Mixtures were centrifuged and the precipitates were washed with cold aceton and dried in vacuo.

Preparation and purification of curdlan anc pachyman. Alcaligenes faecalis var. myxogenes $10 \mathrm{C} 3$ mutant $\mathrm{K}$, was grown in a medium containing $5 \%$ glucose and appropriate amounts of urea and inor ganic salts, at $30^{\circ} \mathrm{C}$ for 5 days. ${ }^{3}$ Then the culturt was centrifuged at $6000 \mathrm{rpm}$ for $30 \mathrm{~min}$. The sedi ments (dry weight, $3 \mathrm{~g}$ ) obtained from four differenı batches of culture were added to $500 \mathrm{ml}$ of $0.5 \mathrm{~N}$ sodium hydroxide at $3^{\circ} \mathrm{C}$, to prevent possible alkaline degradation of the polysaccharide, and the mixture was stirred in a Potter apparatus for $10 \mathrm{~min}$, and then stood for $3 \mathrm{hr}$ at the same temperature. The resulting viscous solution was centrifuged at 13,000 rpm for $30 \mathrm{~min}$, and curdlan in the clear surpernatant was precipitated by neutralization with $10 \%$ acetic acid. This precipitated polysaccharide was repeatedly washed with water, acetone and ether, and then dried in vacuo at $60^{\circ} \mathrm{C}$ (yield, approx. $40 \%$ of glucose present in the culture medium). All preparations (I, II, III and IV) contained $0.21 \sim$ $0.27 \% \mathrm{~N}$, and $93 \sim 94 \%$ glucose.

Pachyman was prepared from air-dried Bukuryo (Poria cocos) purchased from Hisabu Co., Osaka. The powdered fungus $(3 \mathrm{~g})$ was treated with $0.5 \mathrm{~N}$ sodium hydroxide in the cold, as described for isolation of curdlan. Pachyman was precipitated from the alkaline extract by addition of acetic acid, and the gelatinous sediment was washed with water, acetone and ether, and then dried (yield, $77 \%$ of the starting material). To remove traces of contaminating alkali-soluble and water-insoluble impurities, these $\beta-1,3$ glucans were purified further with dimethylsulfoxide (DMSO). Each preparation $(2.5 \mathrm{~g})$ was dissolved in DMSO $(500 \mathrm{ml})$ using a mechanical stirrer at $25^{\circ} \mathrm{C}$ for $15 \mathrm{hr}$. Water $(600 \mathrm{ml})$ was added to the viscous solution and the precipitated polysaccharide was collected by centrifugation. After one more treatment with DMSO and water, the purified polysaccharide was washed thoroughly with water, acetone and ether, and dried (yield: $93 \%$ for curdlan, and 91\% for pachyman).

13) E. T. Reese and M. Mandels, Can. J. Microbiol., 5, 173 (1959). 


\section{RESULTS}

\section{Properties of pachyman}

The purified pachyman which contained $0.02 \% \mathrm{~N}$ was insoluble in water, but it dissolved on gentle agitation in dilute sodium hydroxide to give a solution with $[\alpha]_{D}^{25}+32^{\circ}$ $(c=1.0$ in $1 \mathrm{~N} \mathrm{NaOH})$. Neutralization of the alkaline solution with acid resulted in an insoluble gelatinous precipitate, as in the case of curdlan. Complete hydrolysis of pachyman $(100 \mathrm{mg})$ by treatment at $100^{\circ} \mathrm{C}$ first with $90 \%$ formic acid for $3 \mathrm{hr}$ and then with $1 \mathrm{~N}$ sulfuric acid for $5 \mathrm{hr}$ gave only D-glucose, as shown by paper chromatography (solvents $\mathrm{A}$ and $\mathrm{B}$ ). Its glucose content was $96 \%$, which was comparable to that of the curdlan preparation.

The infrared spectrum of pachyman showed an absorption band at $890 \mathrm{~cm}^{-1}$, characteristic of $\beta$-linked polysaccharide, and was almost identical with that of curdlan."

\section{Gel formation}

As already reported, ${ }^{6)}$ curldan readily gives a firm and resilient gel when heated in aqueous suspension at $90^{\circ} \mathrm{C}$ for $3 \mathrm{~min}$. Pachy-

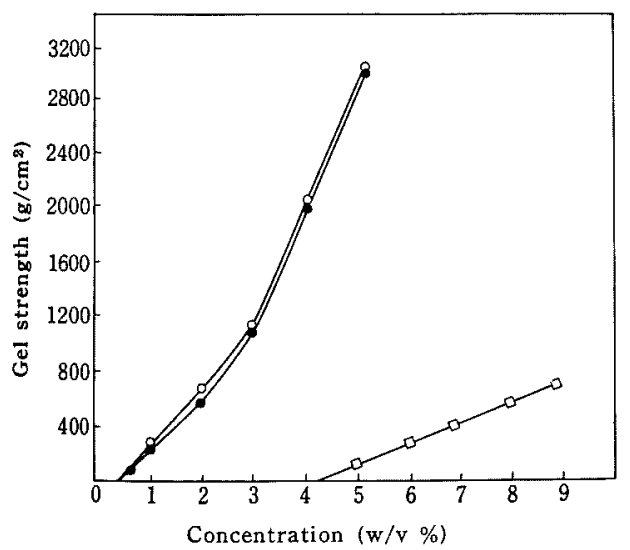

FiG. 1. Comparison of Strengths of Curdlan and Pachyman Gels at Various Concentrations.

Curdlan preparation II (-O-: $\overline{\mathrm{DP}} 455$ ), IV (- - : $\overline{\mathrm{DP}} 135)$ and Pachyman $(-\square-: \overline{\mathrm{DP}} 255)$ were homogenized with $10 \mathrm{ml}$ of water, and heated at $90^{\circ} \mathrm{C}$ for $10 \mathrm{~min}$. After cooling $\left(30^{\circ} \mathrm{C}\right)$ the gel-strengths were measured. man also tended to give a gel on heat treatment, but the minimum concentration required for gel formation was $4.3 \%$, which was much higher than that of curdlan $(0.5 \%)$. Figure 1 shows the relationship of the gelstrength and concentration of curdlan (preparations II and IV) and of pachyman. The gel-strength of all curdlan preparations, even though they had different molecular sizes, was much more than that of pachyman under the same conditions: the former was ca. $350 \mathrm{~g} / \mathrm{cm}^{2}$ with a $1 \%$ suspension, and ca. $3000 \mathrm{~g} / \mathrm{cm}^{2}$ with a $5 \%$ suspension, whilst the latter was only $160 \mathrm{~g} / \mathrm{cm}^{2}$ even with a $5 \%$ suspension. Moreover, the gel formed from pachyman was less elastic than that of curdlan, and readily cracked when pressed.

\section{Degree of polymerization}

The average degree of polymerization $(\overline{\mathrm{DP}})$ of the curdlan preparations (I, II, III and IV) obtained from different batches of culture and of pachyman were estimated by the procedure of Smith et al. ${ }^{14}$ Each sample (60 mg) was dissolved in $0.5 \mathrm{~N}$ sodium hydroxide and reduced by addition of sodium borohydride $(40 \mathrm{mg})$ in the usual way. After complete reduction ( $15 \mathrm{hr}$ ) the excess borohydride was decomposed by addition of acetic acid to $\mathrm{pH} 5.5$, and then the reduced polysaccharide was oxidized with $0.05 \mathrm{M}$ sodium periodate $(20 \mathrm{ml})$ at $25^{\circ} \mathrm{C}$. When oxidation was complete $(48 \mathrm{hr}$ ) the reaction mixture was centrifuged to remove the residual polysaccharide, and aliquots $(2 \mathrm{ml})$ of the supernatant were used for micro-determination of formaldehyde. ${ }^{11 !}$ The amounts of formaldehyde released from $100 \mu$ moles of anhydroglucose units of curdlan preparations I, II, III and IV were on the average of three determinations $0.48,0.44,0.96$ and $1.48 \mu$ moles. These values corresponded to degrees of polymerization of $410,455,210$

14) G. W. Hay, B. A. Lewis, F. Smith and A. M. Unrau, "Methods in Carbohydrate Chemistry," Vol. V, p. 251, Academic Press, 1965. 
and 135, respectively, since 2 moles of formaldehyde should be formed from the original reducing end of the reduced $(1 \rightarrow 3)$-linked glucosidic chain. Therefore, it appears that the degree of polymerization varies between 135 455, depending on the conditions of culture of the microorganism.

Reduced pachyman released $0.78 \mu$ mole of formaldehyde per $100 \mu$ moles of anhydroglucose unit, corresponding to a degree of polymerization of 255 , indicating that the molecular weight of pachyman is similar to that of the curdlan preparation III.

\section{Periodate oxidation}

Preparations (190 mg) of curdlan and pachyman were homogenized with water $(75 \mathrm{ml})$ in a Potter apparatus and oxidized with $0.04 \mathrm{M}$ sodium periodate $(25 \mathrm{ml})$ at $3^{\circ} \mathrm{C}$ for 14 days. At suitable intervals, consumption of periodate and simultaneous production of formic acid were determined. Careful measurement showed that all preparations of curdlan reduced 0.02 mole of periodate/anhydroglucose unit with the production of 0.01 mole of formic acid, whereas under the same conditions, pachyman reduced 0.04 mole of periodate/anhydroglucose unit with the production of 0.02 mole of formic acid. These results indicate that both polysaccharides contain very high proportions of $(1 \rightarrow 3)$ linked glucose residues. However, the pachyman molecule appears to contain relatively more non-reducing ends than the curdlan molecule.

Products of enzymatic hydrolysis and partial acid hydrolysis

Curdlan preparation II and pachyman (100 mg each) were suspended in water $(10 \mathrm{ml})$ and incubated with Rhizopus arrhizus QM1032 enzyme preparation $(10 \mathrm{mg}$ in $5 \mathrm{ml}$ of $0.1 \mathrm{M}$ acetate buffer, $\mathrm{pH} 4.8$ ) at $37^{\circ} \mathrm{C}$ for $3 \mathrm{hr} .{ }^{15 l}$ After centrifugation, the supernatant was evaporated to a small volume $(2 \mathrm{ml})$ and the

15) A. Misaki, J. Johnson, S. Kirkwood, J. V. Scaletti and F. Smith, Carbohyd. Res., 6150 (1968). products were examined by paper chromatography. Digests of curdlan and pachyman contained the same products including glucose, laminaribiose ( $R_{\text {gluc }} 0.73$ in solvent $\mathrm{A}$ ), and a small amount of laminaritriose $\left(R_{\text {gluc }} 0.39\right)$. When incubated with Basidiomycete QM806 enzyme preparation at $\mathrm{pH} 5.0$ for $6 \mathrm{hr},{ }^{16)}$ both curdlan and pachyman produced predominantly glucose with a trace of laminaribiose.

For partial acid hydrolysis, curdlan and pachyman $(200 \mathrm{mg})$ were treated, first with $90 \%$ formic acid at $100^{\circ} \mathrm{C}$ for $2 \mathrm{hr}$ and then with $1 \mathrm{~N}$ sulfuric acid for a further $1.5 \mathrm{hr}$. The hydrolyzate was neutralized with barium carbonate and filtered, and the filtrate was evaporated to a small volume. Paper chromatography (solvents $A$ and $B$ ) revealed spots corresponding to glucose, laminaribiose, laminaritriose and higher $\beta$-(1 $\rightarrow 3)$-linked oligosaccharides ( $R_{\text {gluc }} 0.28$ and 0.17 ). These products were smilar to those obtained by partial hydrolysis of laminarin.

\section{Smith degradation}

Since the data on periodate oxidation and on the degree of polymerization were in favour of the presence of a small proportion of branch points and/or internal $(1 \rightarrow 6)$-glucosidic linkages in the pachyman molecule, the difference between the fine structure of curdlan and pachyman was examined by application of the Smith degradation procedure.

Curdlan preparation II ( $\overline{\mathrm{DP}} 455 ; 4.34 \mathrm{~g}$ ) and pachyman $(4.35 \mathrm{~g})$ were subjected to periodate oxidation with $0.01 \mathrm{M}$ sodium periodate $(600$ $\mathrm{ml}$ ) at $3^{\circ} \mathrm{C}$ in the dark. After 21 days oxidation, each reaction mixture was centrifuged and the oxidized glucan was washed repeatedly with water, and then resuspended in water $(300 \mathrm{ml})$, and reduced with sodium borohydride $(800 \mathrm{mg})$. After stirring at room temperature overnight, more borohydride $(300 \mathrm{mg})$ was added to ensure complete reduction. The excess reagent was decomposed

16) T. E. Nelson, J. V. Scaletti, F. Smith and S. Kirwood, Can. J. Chem., 41, 1671 (1963). 
by neutralization with acetic acid ( $\mathrm{pH} 7.0$ ), and the glucan polyalcohol collected by centrifugation was washed repeatedly with water. Portions of curdlan polyalcohol and pachyman polyalcohol ( $0.35 \mathrm{~g}$, dry weight) were heated with $90 \%$ formic acid $(5 \mathrm{ml})$ in sealed tubes at $100^{\circ} \mathrm{C}$ for $3 \mathrm{hr}$, and then refluxed with $1 \mathrm{~N}$ sulfuric acid for $5 \mathrm{hr}$. Each hydrolyzate was neutralized $\left(\mathrm{BaCO}_{3}\right)$ and filtered, and the filtrate was evaporated to a syrup. Paper chromatography showed two spots, one (major) corresponding to glucose and the other (minor) to glycerol, in the glucan polyalcohols derived from both curdlan and pachyman; erythritol could not be detected. Although in the previous study ${ }^{4}$ ) glycerol could not be detectable in the hydrolyzate of curdlan polyalcohol, this was probably because insufficient amount of material was tested. After separation on chromatograms using solvent A, each component was extracted from the appropriate area of the paper with water $(20 \mathrm{ml})$, and aliquots of the extracts were used for determination of glucose and glycerol. The former was estimated by the phenol-sulfuric acid method, and the latter by the chromotropic acid method. The results showed that the molar ratio of glucose to glycerol was $125: 1$, on the average of three measurements, for the curdlan polyalcohol, and $40: 1$ for the pachyman polyalcohol, corresponding to apparent chain lengths or repeating units of 125 and 40 , respectively.

In another experiment, the bulk of the glucose present in the hydrolyzates was converted to gluconic acid by bromine oxidation, ${ }^{17 /}$ and was removed by passage through an Amberlite IR 120 ( $\mathrm{H}^{+}$-form) column and then a Duolite $\mathrm{A}_{2}\left(\mathrm{OH}^{-}\right.$-form) column (each, $2 \times 20 \mathrm{~cm}$ ), and the neutral eluate was evaporated to a small volume $(2 \mathrm{ml})$. From colorimetric determination of

\footnotetext{
17) H.S. Isbell, "Methods in Carbohydrate Chemistry," Vol. II, p. 13, Academic Press, 1963.
}

the amount of glycerol in solution, the molar proportion of glycerol was $0.77 \%$ in the curdlan polyalcohol, and $2.0 \%$ in the pachyman polyalcohol. The above values corresponded to apparent chain length of 130 , and 50 , respectively.

Most of the samples of curdlan polyalcohol and pachyman polyalcohol ( $3 \mathrm{~g}$, dry weight) were suspended in water $(300 \mathrm{ml})$ and subjected to mild hydrolysis with $0.1 \mathrm{~N}$ sulfuric acid at $25^{\circ} \mathrm{C}$ for $24 \mathrm{hr}$, and the degraded glucans from the curdlan and pachyman were collected by centrifugation as waterinsoluble materials (yield, $2.8 \mathrm{~g}$ from both curdlan and pachyman). Only glycerol could be detected by paper chromatography in the supernatant of the degraded glucans.

\section{Comparison of the degraded glucans from curdlan and pachyman}

When the degraded curdlan and pachyman (100'mg each) were oxidized with $0.01 \mathrm{M}$ sodium periodate at $3^{\circ} \mathrm{C}$, in the same way as the native glucans, the degraded curdlan reduced 0.02 mole of periodate/anhydroglucose unit with simultaneous production of 0.01 mole of formic acid, whereas the degraded pachyman reduced 0.04 mole of periodate/ anhydroglucose unit with production of 0.02 mole of formic acid. These results suggest that the basic structural features of curdlan and pachyman were not altered by Smith degradation.

The degraded curdlan and pachyman (350 mg each) were completely hydrolyzed by treatment with formic acid and $1 \mathrm{~N}$ sulfuric acid, as described previously. Paper chromatography (solvents $A$ and $B$ ) revealed the presence of glycerol together with glucose (major component). The ratio of glucose to glycerol was approximately $190: 1$ in the degraded curdlan, and 210:1 in the degraded pachyman. Since Smith degradation cleaves the non-reducing ends of the molecule with liberation of glycerol, the glycerol present in the hydrolyzate of the residual (degraded) polysaccharide must arise from the 3-O-sub- 
stituted glycerol originating from internal $\beta$ - $(1 \rightarrow 6)$-glucosidic linkages.

3-O-Substituted glycerol gives one mole of formaldehyde on periodate oxidation, so the apparent degree of polymerization of the degraded glucans can be calculated. When the degraded glucans ( $70 \mathrm{mg}$ each) were oxidized with $0.05 \mathrm{M}$ sodium periodate $(20 \mathrm{ml})$ at $25^{\circ} \mathrm{C}$ for $48 \mathrm{hr}$, the degraded curdlan and degraded pachyman released $0.65 \mu$ mole and $0.75 \mu$ mole of formaldehyde per $100 \mu$ moles of anhydroglucose units, respectively. The above values correspond to degrees of polymerization of approximately 155 for degraded curdlan and 130 for degraded pachyman.

The following experiment was carried out to see if Smith degradation caused any hydrolytic cleavage of $\beta$-(1 $\rightarrow 3)$-glucosidic linkage. The degraded glucans ( $1 \mathrm{~g}$ each) were subjected to periodate oxidation, borohydride reduction, and mild hydrolysis, as described above. If mild acid treatment causes hydrolysis of the $(1 \rightarrow 3)$-linked glucose residues, the reducing terminal units of the resulting degraded glucans should give rise to formaldehyde upon oxidation with periodate. After re-treatment by the Smith degradation procedure, the degraded glucans derived both from curdlan and pachyman did not produce any appreciable amount of formaldehyde when oxidized with $0.05 \mathrm{~m}$ sodium periodate at $25^{\circ} \mathrm{C}$ for $48 \mathrm{hr}$. This clearly indicates that, under the conditions adopted, little or no cleavage of the $\beta-(1 \rightarrow 3)$-linkage occurs.

Comparison of the degrees of polymerization of degraded curdlan, 155, and native curdlan, 455 , indicates that two internal $(1 \rightarrow 6)$-glucosidic linkages are most probably present in the latter. Similarly, pachyman ( $\overline{D P}$ 255) was shown to contain one such periodatesensitive internal linkage in the molecule, since Smith degradation caused cleavage of the chain to produce a degraded glucan with $\overline{\mathrm{DP}}$ 130. These results also indicate that there is little or no branching in the curdlan molecule, because the degree of polymeriza-
Table I. Properties of Curdlan AND PACHYMAN

$\begin{array}{lcc}\text { Property } & \text { Curdlan } & \text { Pachyman } \\ {[\alpha]_{\mathrm{D}}^{25}(c=1,1 \mathrm{~N} \mathrm{NaOH})} & +18^{\circ} & +32^{\circ} \\ \begin{array}{c}\text { Infrared absorption } \\ \text { peak }\left(\mathrm{cm}^{-1}\right)\end{array} & 890 & 890 \\ \begin{array}{c}\text { Strength of gel from } 5 \% \\ \quad \text { suspension (g/cm }{ }^{2} \text { ) }\end{array} & 3000 & 160 \\ \begin{array}{c}\text { Consumption of periodate } \\ \text { (mole/anhydroglucose unit) }\end{array} & 0.02 & 0.04 \\ \begin{array}{c}\text { Production of formic acid } \\ \text { (mole/anhydroglucose unit) }\end{array} & 0.01 & 0.02 \\ \begin{array}{c}\text { Degree of polymerization } \\ \text { Apparent chain length }\end{array} & 455^{\mathrm{a})} & 255 \\ \end{array}$

a) Curdlan preparation II.

tion of the degraded glucan is consistent with the apparent chain length $(125 \sim 130)$ of the native glucan, as estimated from the content of glycerol in the curdlan polyalcohol. By contrast, pachyman ( $\overline{\mathrm{DP}} 255$ ) probably contains four branch points, in addition to an internal $(1 \rightarrow 6)$-linkage, since the degree of polymerization of the degraded glucan was 130 and the average chain length of the native glucan was 40 .

The properties of curdlan and pachyman revealed by the present investigation are shown in Table I.

\section{DISCUSSION}

In the previous study, the isolation of 2,4,6-tri-O-methyl-D-glucose and a very small amount of 2,3,4,6-tetra-O-methyl-D-glucose, and the resistance to periodate oxidation proved that curdlan is an essentially linear molecule built up of $\beta$-(1-3)-linked glucose units. This polysaccharide is not soluble in water, but when the aqueous suspension is heated it gives a firm and resilient gel. ${ }^{61}$ Since the long linear $\beta-(1 \rightarrow 3)$-linked chain appeared to be responsible for these peculiar physical properties, it was thought of interest to compare its rheological characters with those of other $\beta-1,3$ glucans. Among the $\beta$ glucans containing exclusively $(1 \rightarrow 3)$-linked glucose residues, pachyman from Bukuryo (Poria cocos) was chosen, because it is com- 
mercially available and its structure may resemble that of curdlan, as shown by the earlier work of Wari and Whelan. ${ }^{5}$

In the present study, both curdlan and pachyman were purified with dimethylsulfoxide, which dissolves both the glucans. The purified pachyman had $[\alpha]_{\mathrm{D}}^{25}+32^{\circ}$ in alkali, and showed an infrared spectrum almost identical to that of curdlan. The endo- $\beta-1,3$ glucanase from Rhizopus arrhizus QM1032 hydrolyzed both curdlan and pachyman with production of the same sugars, e.g. glucose, laminaribiose and laminaritriose. Although gentiobiose could not be detected by paper chromatography, the results obtained by Smith degradation showed that there were probably a few $\beta$-(1 $\rightarrow 6)$-glucosidic linkages in both curdlan and pachyman. Exo$\beta-1,3$ glucanase also hydrolyzed both polysaccharides to glucose.

The degree of polymerization of the curdlan preparations obtained from the different batches of culture varied between 135 and 455. The difference between different preparations is perphaps due to differences in conditions of culture. The degree of polymerization of pachyman, 255, is not significantly different from the average value of that of curdlan. The main structure of pachyman is, therefore, very similar to that of curdlan (see, Table I).

Due to their physical natures, both polysaccharides are insoluble in water but soluble in dilute alkali. However, gel formation from pachyman and curdlan differed. Although pachyman gave a gel like curdlan on heating in aqueous suspension, the minimum concentration required for gel-formation was much higher than with curdlan. Moreover, the strength of the gel from pachyman was much lower than that from curdlan (see Fig. 1). These findings prompted us to compare the fine structure of the two, and particularly to investigate for the possible presence of minor linkages in pachyman, such as internal $(1 \rightarrow 6)$-linkages or branch points, such as those present in most known $\beta-1,3$ glucans, e.g. laminarin, leucosin and yeast glucan.

Oxidation of curdlan with sodium periodate at low temperature resulted in consumption of 0.02 mole of periodate/anhydroglucose unit with the production of 0.01 mole of formic acid. These values are lower than those obtained previously on oxidation at $23^{\circ} \mathrm{C}$. . $^{\prime}$ This difference may be because at low temperature formic acid is produced only from the non-reducing ends of $\beta-(1 \rightarrow 3)$-linked glucan, since the formyl ester formed at the reducing end would be hydrolyzed very slowly, whereas at room temperature formic acid is also produced from the reducing end. ${ }^{121}$

Pachyman reduced 0.04 mole of periodate/ anhydroglucose unit with production of 0.02 mole of formic acid, suggesting that it contains more "triol" groups (i.e. non-reducing ends and/or internal $(1 \rightarrow 6)$-glucosidic linkages) than curdlan.

Detailed information on the structure of curdlan and pachyman was obtained by examination of the products of Smith degradation. The curdlan preparation II with $\overline{\mathrm{DP}}$ 455 was subjected to periodate oxidation at low temperature, and the resulting oxidized glucan was reduced with sodium borohydride to the corresponding polyalcohol. Complete hydrolysis with $90 \%$ formic acid and then $1 \mathrm{~N}$ sulfuric acid yielded glucose and a small amount of glycerol. If curdlan contained only $\beta-(1 \rightarrow 3)$-glucosidic linkages, glycerol must arise from the non-reducing end. If however, an internal $(1 \rightarrow 6)$-linkage is present, as shown in (I), then additional glycerol should be released by complete hydrolysis. The amount of glycerol released from the curdlan polyalcohol corresponded to one "triol" group per 125 130 glucose units, indicating that these are two internal $(1 \rightarrow 6)$ glucosidic linkages in the curdlan molecule with $\overline{\mathrm{DP}} 455$. On mild hydrolysis with $0.1 \mathrm{~N}$ sulfuric acid at $25^{\circ} \mathrm{C}$ the curdlan polyalcohol gave the degraded glucan in $93 \%$ yield, with 


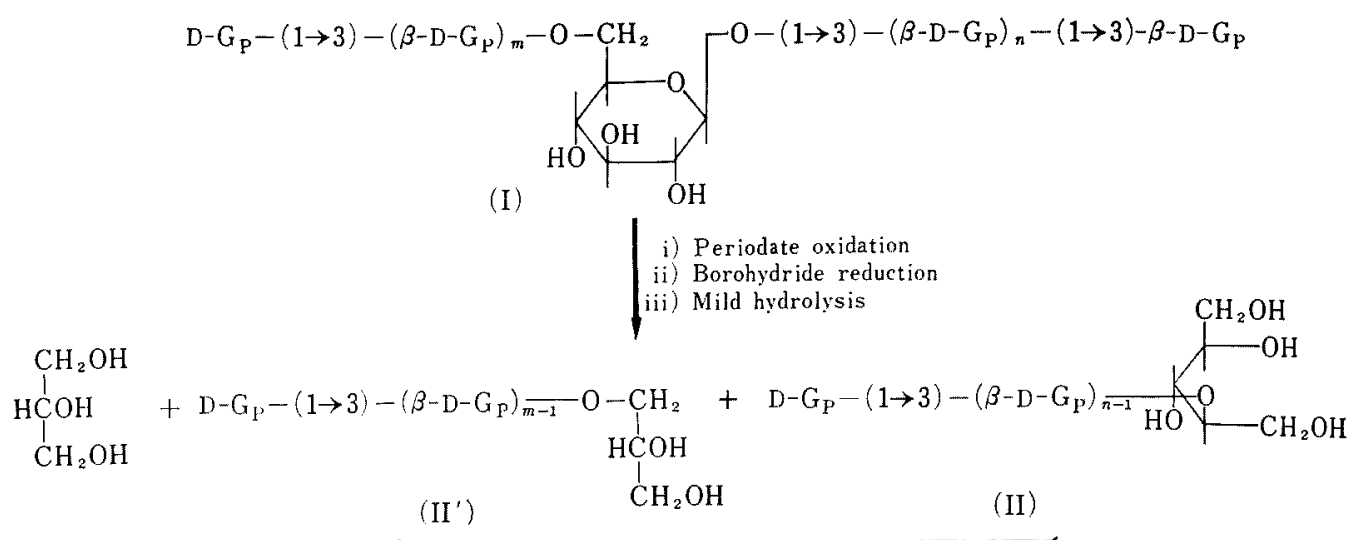

i) Periodate oxidation

ii) Borohydride reduction

iii) Mild hydrolysis

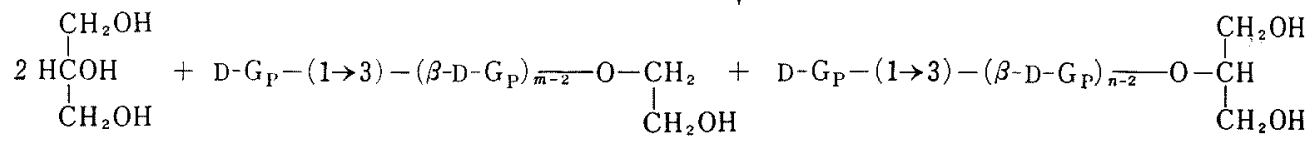

(III')

(III)

(D-G $\mathrm{G}_{P}: \mathrm{D}-\mathrm{Glucopyranose}$ residue)

SCHEME 1. Smith Degradation of $\beta-1,3$ Glucan Containing an Internal (1 $\rightarrow 6)$-Glucosidic Linkage.

simultaneous liberation of a trace of glycerol, probably from the non-reducing end. If the parent glucan contains only $(1 \rightarrow 3)$-linkages, mild acid treatment should not cause hydrolysis of the chain, and hence, on periodate oxidation only one mole of formaldehyde would be released from the product of mild acid hydrolysis, assuming that the chain is terminated by a 2-O-substituted D-arabitol residue $^{181}$ (II). The degraded glucan derived from the curdlan polyalcohol released a small but significant amount of formaldehyde during oxidation, indicating the presence of a 3 -substituted glycerol residue $\left(\mathbf{I I}^{\prime}\right)$. The fact

18) E. Percival, R. H. McDowell, "Chemistry and Enzymology of Marine Algal Polysaccharides," p. 60, Academic Press, 1967. that after re-treatment by Smith degradation the degraded glucans (III and III') released $^{\prime}$ only neglegible amounts of formaldehyde indicates that no cleavage of $\beta-(1 \rightarrow 3)$-linkages occurred during mild hydrolysis. The release of formaldehyde corresponded to a degree of polymerization of 155 . This result, together with the $\overline{\mathrm{DP}} 455$ of the native glucan, strongly suggests that two internal $(1 \rightarrow 6)$ glucosidic linkages are present in curdlan. Since the $\overline{\mathrm{DP}}$ value of the degraded curdlan is consistent with the apparent chain of the native curdlan, $125 \sim 130$, it is evident that the curdlan molecule is essentially linear with no branching.

Complete hydrolysis of the pachyman polyalcohol yielded one mole of glycerol per approximately 40 glucose units, corresponding 
to six "triol" groups including non-reducing ends and internal $(1 \rightarrow 6)$-linkages in the molecule with $\overline{\mathrm{DP}} 255$. Since the degraded glucan obtained by Smith degradation had a degree of polymerization of 130, pachyman appears to contain approximately four branch points besides one internal $(1 \rightarrow 6)$-glucosidic linkage. This branched structure is consistent with the recent result of Smith and Handa, who re-examined the structure of pachyman by methylation analysis and provided evidence for the presence of a few $(1 \rightarrow 6)$-glucosidic branch points (N. Handa, personal communication).

The present investigation leads to the conclusion that both curdlan and pachyman may contain a few internal $(1 \rightarrow 6)$-glucosidic linkages. However, the significant difference between the two polysaccharides is that the former is linear, but the latter has a few branch points, one for every 50 glucose residues. The difference between gel formation by curdlan and pachyman may be explained by the presence of branches in the pachyman molecule. In this connection, it is interesting that soluble laminarins were shown by Fleming et al..$^{19 \prime}$ to contain $2 \sim 3$ branch points, in contrast to insoluble laminarins which are essentially linear.

The solubilities of linear $\beta-1,3$ glucans are known to be related to the degree of their polymerization. Laminarin (soluble form, $\overline{\mathrm{DP}} 36)^{201}$ is soluble both in water and alkali, whereas paramylons from Peranema trichophorum $(\overline{\mathrm{DP}} 80)^{211}$ and from Euglena gracilis

19) M. Fleming and D. J. Manners, Biochem. $J$, 94, 17 (1965)

20) A. Beattie, E. L. Hirst and E. Percival, Biochem. J., 79, 531 (1961).

21) A. R. Archibald, W. L. Cunningham, D.J. Manners and J. R. Stark, ibid., 88, 444 (1963).
$(\overline{\mathrm{DP}} 150)^{22}$ are insoluble in water but soluble in alkali. Curdlan and pachyman both of which are insoluble in water but soluble in alkali may resemble the paramylon of Euglena gracilis. In addition to their solubility characters, it is now apparent that long $\beta$ $(1 \rightarrow 3)$-glucosidic linked chains form firm, resilient gels on heating in aqueous suspension. Yeast glucan is long known to be a $\beta-1,3$ glucan. However, it is insoluble both in water and alkali, and does not form a gel on heat treatment. These different properties may be attributed to its branched structure, ${ }^{15,23)}$ in which numerous $\beta-(1 \rightarrow 3)$-linked side chains are attached to the $\beta-(1 \rightarrow 6)$-linked main chain to afford a stable framework through hydrogen bonds.

The presence of $(1 \rightarrow 6)$-linkages in the curdlan molecule is interesting, since succinoglucan $^{24)}$ produced by the parent strain, Alcaligenes faecalis var. myxogenes 10C3, was found to contain approximately $10 \%$ of internal $\beta$-( $1 \rightarrow 6)$-glucosidic linkages, in addition to $\beta-(1 \rightarrow 3)$ - and $\beta$-(1 $1 \rightarrow 4)$-glucosidic linkages (unpublished result).

Acknowledgements. The authors thank Professor S. Kirkwood of University of Minnesota for supplying strains of Rhizopus arrhizus QM1032 and Basidiomycete QM806. This work was supported by a research grant from the Ministry of Education.

22) A. E. Glarke and B. A. Stone, Biochim. Biophys. Acta, 44, 161 (1960) [also see, Rev. Pure Appl. Chem., 13, 134 (1963)].

23) D. J. Manners and J.C. Patterson, Biochem. $J ., 98,19 \mathrm{C}$ (1966).

24) T. Harada, Arch. Biochem. Biophys., 112, 65 (1965). 